\title{
AUTOMORPHISMS OF THE UNIT GROUPS OF SQUARE RADICAL ZERO FINITE COMMUTATIVE COMPLETELY PRIMARY RINGS
}

\author{
Ojiema M. Onyango ${ }^{1}$, Owino M. Oduor ${ }^{2}$, Odhiambo P. Oleche $^{3}$ \\ ${ }^{1}$ Department of Mathematics \\ Masinde Muliro University of Science and Technology \\ P.O. Box 190-50100, Kakamega, KENYA \\ ${ }^{2}$ Department of Mathematics and Computer Science \\ University of Kabianga \\ P.O. Box 2030-20200, Kericho, KENYA \\ ${ }^{3}$ Department of Pure and Applied Mathematics \\ Maseno University \\ P.O. Box 333, Maseno, KENYA
}

\begin{abstract}
Let $G$ be a group. The groups $G^{\prime}$ for which $G$ is an automorphism group have not been fully characterized. Suppose $R$ is a Completely Primary finite Ring with Jacobson Radical $J$ such that $J^{2}=(0)$. In this case, the characteristic of $R$ is $p$ or $p^{2}$ and the group of units $R^{*}=\mathbb{Z}_{p^{\mathrm{r}}-1} \times(I+J)$. The structure of $R^{*}$ is well known, but its automorphism group is not well documented. Given the group $R^{*}$, let $\operatorname{Aut}\left(R^{*}\right)$ denote the group of isomorphisms $\phi: R^{*} \rightarrow R^{*}$ with multiplication given by the composition of functions. The structure of the automorphism groups of finite groups is intimately connected to the structure of the finite groups themselves. In this note, we determine the structure of $\operatorname{Aut}\left(R^{*}\right)$ using well known procedures and to this end, extend the results previously obtained in this area of research.
\end{abstract}

AMS Subject Classification: 20K30, 16P10

Key Words: automorphism groups, unit groups, square radical zero, completely primary rings

Received: February 4, 2016

Published: May 28, 2016 (c) 2016 Academic Publications, Ltd. url: www.acadpubl.eu 


\section{Introduction}

The definition of terms and standard notations can be obtained from $[2,3,5,6]$. The classification of finite rings has been studied with great success in the recent past $([2,5,6]$ and related studies). Most of the researchers have concentrated in obtaining the structures of the unit groups of Completely Primary finite rings. However, the automorphisms of these unit groups have remained uncharacterized. The first general structure result for the automorphisms group of a finite group follows from a classical result of Gauss in number theory. Let $\mathbb{Z}_{n}$ denote the additive group of integers $\bmod n$ and $U\left(\mathbb{Z}_{n}\right)$ the multiplicative group of integers $\bmod n$. Gauss analyzed the orders of elements in $U\left(\mathbb{Z}_{p^{n}}\right)$ for $p$ prime. His results can be summarized as follows:

Theorem 1. (Gauss) Let $p$ be an odd prime and $n \geq 1$ or $p=2$ and $n \geq 2$. Then

$$
U\left(\mathbb{Z}_{p^{n}}\right) \cong \mathbb{Z}_{p^{n-1}(p-1)}, U\left(\mathbb{Z}_{2^{n}}\right) \cong \mathbb{Z}_{2} \times \mathbb{Z}_{2^{n-2}}
$$

Notice that $U\left(\mathbb{Z}_{n}\right)$ is precisely the set of generators of $\mathbb{Z}_{n}$. Since any automorphism $\theta \in \mathbb{Z}_{n}$ sends 1 to a generator, the valuation map $E: \operatorname{Aut}\left(\mathbb{Z}_{n}\right) \mapsto$ $U\left(\mathbb{Z}_{p^{n}}\right)$ given by $E(\theta)=E(1)$ is an isomorphism of groups. This sets the stage for prime factorization of the integer $n$ and consequently the classification of the automorphisms of an arbitrary finite abelian group. On the other hand, the automorphisms of cyclic groups are precisely known. In fact, given any prime $p$ and any integer $n$, the group $\operatorname{Aut}\left(C_{p}^{n}\right) \cong \operatorname{Aut}\left(\mathbb{Z}_{p}^{n}\right)$, the group of $n$ by $n$ invertible matrices over the field $\mathbb{Z}_{p}$. These and related matrix groups play important roles in the classification of simple groups.

In [5], we constructed a class of Square Radical Zero Commutative Completely Primary finite Rings as follows:

Let $R_{o}$ be the Galois ring of the form $G R\left(p^{k r}, p^{k}\right)$, such that $k=1,2$. For each $i=1, \ldots, h$, let $u_{i} \in J(R)$, such that $U$ is an $h$-dimensional $R_{o}$-module generated by $\left\{u_{1}, \ldots, u_{h}\right\}$ so that $R=R_{0} \oplus U$ is an additive group. On this group, define multiplication by the following relation

$$
p u_{i}=u_{i} u_{j}=u_{j} u_{i}=0, \quad u_{i} r_{o}=\left(r_{o}\right)^{\sigma_{i}} u_{i}
$$

where $r_{o} \in R_{o}, 1 \leq i, j \leq h, p$ is a prime integer, $n$ and $r$ are positive integers and $\sigma_{i}$ is the automorphism associated with $u_{i}$. Further, let the generators $\left\{u_{i}\right\}$ for $U$ satisfy the additional condition that, if $u_{i} \in U$, then, $p u_{i}=u_{i} u_{j}=0$. From the given multiplication in $R$, we see that if $r_{0}+\sum_{i=1}^{h} \lambda_{i} u_{i}$ and $s_{0}+\sum_{i=1}^{h} \gamma_{i} u_{i}$, 
$r_{0}, s_{0} \in R_{0}, \lambda_{i}, \gamma_{i} \in F_{o}$ are elements of $R$, then,

$$
\left(r_{o}+\sum_{i=1}^{h} \lambda_{i} u_{i}\right)\left(s_{o}+\sum_{i=1}^{h} \gamma_{i} u_{i}\right)=r_{o} s_{o}+\sum_{i=1}^{h}\left\{\left(r_{o}+p R_{o}\right) \gamma_{i}+\lambda_{i}\left(s_{o}+p R_{o}\right)^{\sigma_{i}}\right\} u_{i} .
$$

It is easy to verify that the given multiplication turns the additive abelian group $R$, into a ring with identity $(1,0, \ldots, 0)$. Moreover, $J^{2}=(0)$. Accordingly, the characteristic of $R$ is either $p$ or $p^{2}$. Furthermore, the group of units $R^{*}$ of $R$ is given by $R^{*}=\mathbb{Z}_{p^{r}-1} \times(1+J)$, a direct product of abelian groups.

In [3], Hillar and Rhea have given a useful description of the automorphism group of an arbitrary finite abelian group and they found the size of this automorphism group. We extend their work by characterizing $\operatorname{Aut}\left(R^{*}\right)$. We find all the elements of $G L_{h r}\left(\mathbb{Z}_{p}\right)$ that can be extended to a matrix in $\operatorname{End}\left(B_{p}\right)$ and calculate the distinct ways of extending such elements to the endomorphism. The first complete characterization of the automorphism group of an abelian group was however given by Ranum [1].

\section{Preliminaries}

Theorem 2. (cf. [5]) The unit group $R^{*}$ of the commutative completely primary finite ring of characteristic $p$ or $p^{2}$ with maximal ideal $J$ such that $J^{2}=(0)$ and with invariants $p$ (prime integer), $p \in J, r \geq 1$ and $h \geq 1$ is a direct product of cyclic groups as follows:

(i) If Char $R=p$, then

$$
R^{*}=\mathbb{Z}_{p^{r}-1} \times\left(\mathbb{Z}_{p}^{r}\right)^{h}
$$

(ii) If Char $R=p^{2}$, then

$$
R^{*}=\mathbb{Z}_{p^{r}-1} \times \mathbb{Z}_{p}^{r} \times\left(\mathbb{Z}_{p}^{r}\right)^{h}
$$

The following lemma will be useful in the sequel.

Lemma 1. (cf. [3]) Let $H$ and $K$ be finite groups of relatively prime orders. Then, $\operatorname{Aut}(H) \times \operatorname{Aut}(K) \cong \operatorname{Aut}(H \times K)$

Remark 1. Since the groups $\mathbb{Z}_{p^{r}-1}$ and $\left(\left(\mathbb{Z}_{p}^{r}\right)\right)^{h}$ are of relatively prime orders and $\operatorname{Aut}\left(\mathbb{Z}_{p^{r}-1}\right) \cong\left(\mathbb{Z}_{p^{r}-1}\right)^{*}$, it implies that, when $\operatorname{char}(R)=p$ then

$$
\operatorname{Aut}\left(R^{*}\right) \cong \operatorname{Aut}\left(\mathbb{Z}_{p^{r}-1}\right) \times \operatorname{Aut}\left(\left(\mathbb{Z}_{p}^{r h}\right)\right) \cong\left(\mathbb{Z}_{p^{r}-1}\right)^{*} \times \operatorname{Aut}\left(\left(\mathbb{Z}_{p}^{r h}\right)\right) .
$$

Similarly, when $\operatorname{char}(R)=p^{2}$, then

$$
\operatorname{Aut}\left(R^{*}\right) \cong\left(\mathbb{Z}_{p^{r}-1}\right)^{*} \times \operatorname{Aut}\left(\left(\mathbb{Z}_{p}^{r(h+1)}\right)\right) .
$$


Lemma 2. Let $C h a r(R)=p, p$ a prime integer and

$$
B_{p}=1+J=\left(\mathbb{Z}_{p}^{r}\right)^{h} .
$$

Then, $\left|B_{p}\right|=p^{r h}$.

Proof. If $G=\prod_{i=1}^{r} \mathbb{Z} / p^{e_{i}} \mathbb{Z}$ such that $1 \leq e_{1} \leq e_{2} \leq \ldots \leq e_{r}$, then

$$
\left|\prod_{i=1}^{r} \mathbb{Z} / p^{e_{i}} \mathbb{Z}\right|=p^{\sum_{i=1}^{r} e_{i}}
$$

Now, in $B_{p}, e_{1}=e_{2}=\ldots=e_{r}=1$ and there are $h$-tuples of such $r$ factors of $e_{i}$, so that $\left(\sum_{i=1}^{r} e_{i}\right) h=r h$. Thus $\left|B_{p}\right|=p^{r h}$ as required.

Remark 2. Now, suppose $G \cong \prod_{i}^{h} B_{p}^{\prime}$ such that

$$
B_{p}^{\prime}=\underbrace{\mathbb{Z}_{p} \times \mathbb{Z}_{p} \times \ldots \times \mathbb{Z}_{p}}_{r}
$$

over distinct set of primes $p$, then $\operatorname{Aut}(G)=\prod \operatorname{Aut}\left(B_{p}^{\prime}\right)$.

In the sequel, we determine $\operatorname{Aut}(I+J)$ for both the characteristics of $R$. Suppose $M_{p}=\operatorname{End}\left(B_{p}\right)$, then, the elements of $M_{p}$ are group homomorphisms $\alpha: B_{p} \rightarrow B_{p}$ with ring multiplication given by composition and addition taken naturally. These rings behave like matrix rings.

\section{The Endomorphism Ring and its Properties}

\subsection{Characteristic of $R=p$}

Proposition 1. Let $R$ be a finite ring whose additive group $(R,+)$ is of type $\left(p^{e_{1}}, p^{e_{2}} \ldots, p^{e_{l}}\right): e_{i} \geq e_{2} \geq \ldots \geq e_{l}$. Then, $R$ can be identified with a subring of the endomorphism ring say $B$ of the additive group. The ring $B$ can be considered as the ring of all $l \times l$ matrices $\left(a_{i j}\right)$ such that $1 \leq i, j \leq l$ of the form.

$$
\left(a_{i, j}\right)=\left(\begin{array}{cccc}
a_{11} & a_{12} & \cdots & a_{1 l} \\
p^{e_{1}-e_{2}} a_{21} & a_{22} & \cdots & a_{2 l} \\
\vdots & \vdots & \vdots & \vdots \\
p^{e_{1}-e_{2}} a_{l 1} & \cdots & \cdots & a_{l l}
\end{array}\right)
$$


such that

$$
a_{i j}= \begin{cases}a_{i j}, & i \leq j \\ p^{e_{j}-e_{i}} a_{i j}, & i>j\end{cases}
$$

Proposition 2. Let

$$
1+J=B_{p}=\underbrace{\mathbb{Z}_{p}^{r} \times \mathbb{Z}_{p}^{r} \times \ldots \times \mathbb{Z}_{p}^{r}}_{h}
$$

and $B_{p}^{\prime}=\mathbb{Z}_{p}^{r}$. We define the endomorphism ring of $B_{p}^{\prime}$ by

$$
\operatorname{End}\left(B_{p}^{\prime}\right)=\left\{\left(a_{i j}\right) \in \mathbb{Z}_{r \times r}: p^{e_{i}-e_{j}} \mid a_{i j} ; 1 \leq j \leq i \leq r\right\} .
$$

Example 1. If $n=4$ and since $e_{1}=e_{2}=e_{3}=e_{4}=1$, it follows that if $a_{i j} \in \operatorname{End}\left(B_{p}^{\prime}\right)$ satisfies the conditions of the above proposition, that is

$$
p^{e_{i}-e_{j}} \mid a_{i j} ; 1 \leq j \leq i \leq r
$$

then $a_{i j} \equiv 0(\bmod p) \forall i, j \leq 4$. Suppose $M_{p}^{\prime}=\operatorname{End}\left(B_{p}^{\prime}\right)$, then

$$
M_{p}^{\prime}=\left(\begin{array}{llll}
a_{11} & a_{12} & a_{13} & a_{14} \\
a_{21} & a_{22} & a_{23} & a_{24} \\
a_{31} & a_{32} & a_{33} & a_{34} \\
a_{41} & a_{42} & a_{43} & a_{44}
\end{array}\right)
$$

Thus, generally,

$$
M_{p}^{\prime}=\left(\begin{array}{cccc}
a_{11} & a_{12} & \cdots & a_{1 r} \\
a_{21} & a_{22} & \cdots & a_{2 r} \\
\vdots & \vdots & \vdots & \vdots \\
a_{r 1} & a_{r 2} & \cdots & a_{r r}
\end{array}\right)=\operatorname{End}\left(B_{p}^{\prime}\right)
$$

Proposition 3. Consider $B_{p}^{\prime}=\underbrace{\mathbb{Z}_{p} \times \ldots \times \mathbb{Z}_{p}}_{r}$ as defined above. Then:

(i) $\operatorname{End}\left(B_{p}^{\prime}\right)=M_{r}\left(\mathbb{Z}_{p}\right)$;

(ii) $\operatorname{Aut}\left(B_{p}^{\prime}\right)=G L_{r}\left(\mathbb{Z}_{p}\right)$;

(iii) $\left|\operatorname{Aut}\left(B_{p}^{\prime}\right)\right|=\prod_{i=0}^{r-1}\left(p^{r}-p^{i}\right)$. 
Proof. The proof of (i) and (ii) follow from the previous proposition.

Now, consider $A u t(\underbrace{\mathbb{Z}_{p} \times \ldots \times \mathbb{Z}_{p}}_{r})$. We start with $\operatorname{Aut}\left(\mathbb{Z}_{p}\right)$ and $\operatorname{Aut}\left(\mathbb{Z}_{p} \times \mathbb{Z}_{p}\right)$ in order to obtain the size of the automorphism group of $B_{p}^{\prime}$. In $\mathbb{Z}_{p}$, each of the $p-1$ nonidentity elements has order $p$. Suppose $\mathbb{Z}_{p}=<a>$, then the map $a \mapsto a^{i}$ is an element of $A u t\left(\mathbb{Z}_{p}\right)$ provided $i \in[1, p-1]$. Thus $\left|A u t\left(\mathbb{Z}_{p}\right)\right|=$ $p-1=\Phi(p)$, where $\Phi$ is the Eulers'-phi function.

Next, let $a$ and $b$ each generate groups of order $p$, so that $\mathbb{Z}_{p} \times \mathbb{Z}_{p}=<$ $a><b>$. A homomorphism $\theta: \mathbb{Z}_{p} \times \mathbb{Z}_{p} \mapsto \mathbb{Z}_{p} \times \mathbb{Z}_{p}$ is an automorphism iff $|\theta(a)|=|\theta(b)|=p$ and $\langle\theta(a)>$ intersects with $\langle\theta(b)>$ only at identity.

To find $\left|\mathbb{Z}_{p} \times \mathbb{Z}_{p}\right|$, we must count the pairs $\left(\beta, \beta^{\prime}\right)$ of elements in $\mathbb{Z}_{p} \times \mathbb{Z}_{p}$ such that $\theta(a)=\beta$ and $\theta(b)=\beta^{\prime}$ determines an automorphism. Each of the $p^{2}-1$ nonidentity elements of $\mathbb{Z}_{p} \times \mathbb{Z}_{p}$ has order $p$, so, a given element of $\operatorname{Aut}\left(\mathbb{Z}_{p} \times \mathbb{Z}_{p}\right)$ may map $a$ to any of the $p^{2}-1$ different places.

Let $\beta$ be nonidentity element. We must count the elements $\beta^{\prime}$ of $\mathbb{Z}_{p} \times \mathbb{Z}_{p}$ such that $\beta^{\prime}=p$ and $\langle\beta\rangle \cap\left\langle\beta^{\prime}\right\rangle=\{e\}$. Since each $\beta$ generates a group of order $p$ and any of the $p^{2}-p$ elements of $\mathbb{Z}_{p} \times \mathbb{Z}_{p}$ lying outside of $\langle\beta\rangle$ will generate a group of order $p$ that intersects the group $\langle\beta\rangle$ only at identity element, it follows that

$$
\left|A u t\left(\mathbb{Z}_{p} \times \mathbb{Z}_{p}\right)\right|=\left(p^{2}-1\right)\left(p^{2}-p\right)
$$

For $B_{p}^{\prime}=\underbrace{\mathbb{Z}_{p} \times \ldots \times \mathbb{Z}_{p}}_{r}$, let $\left\{g_{1}, \ldots, g_{r}\right\}$ be a set of generators for $B_{p}^{\prime}$, so that

$$
\underbrace{\mathbb{Z}_{p} \times \ldots \times \mathbb{Z}_{p}}_{r}=<g_{1}>\times<g_{2}>\times \ldots \times<g_{r}>
$$

Each of the nonidentity elements of $B_{p}^{\prime}$ has order $p$. We now count the number of injective maps from the above generators to nonidentity elements that generate groups intersecting only at the identity element.

Suppose that an automorphism of $B_{p}^{\prime}$ sends $g_{1}$ to some element $\beta$ in $B_{p}^{\prime}$, then there are $p^{r}-p$ elements $\beta^{\prime}$ such that $\left\langle\beta>\cap<\beta^{\prime}>\right.$. Supposing further that this automorphism is given by $g_{1} \mapsto \beta$ and $g_{2} \mapsto \beta^{\prime}$ for some $\beta^{\prime}$ not in $\langle\beta>$, there remain $p^{r}-p^{2}$ elements $\beta^{\prime \prime} \in B_{p}^{\prime}$ that are outside of $\langle\beta\rangle \times\left\langle\beta^{\prime}>\right.$. Sending $g_{3}$ to any such $\beta^{\prime \prime}$ gives $\left(<\beta><\beta^{\prime}>\right) \cap<\beta^{\prime \prime}>=\{e\}$.

Continuing in this manner, it is easy to specify where an automorphism of $B_{p}^{\prime}$ sends the first $n$ generators and then find $p^{r}-p^{n}$ elements in $B_{p}^{\prime}$ to which 
the next generators might be sent. Thus

$$
\left|\operatorname{Aut}\left(\prod_{i=1}^{r} \mathbb{Z}_{p}\right)\right|=\left|\operatorname{Aut}\left(B_{p}^{\prime}\right)\right|=\prod_{i=0}^{r-1}\left(p^{r}-p^{i}\right)
$$

Before stating our main results, we need the following properties:

From [3], we have $H_{p}$ as a form of $B_{p}$ thus $B_{p}$ forms a ring. by

Let $\theta_{i}: \mathbb{Z} \mapsto\left(\mathbb{Z}_{p}^{r}\right)^{h}$ defined by $\theta(x)=x(\bmod p)$ be a homomorphism given

$$
\theta\left(x_{11}, \ldots, x_{h r}\right)=\theta_{1}\left(x_{11}\right) \ldots \theta_{h r}\left(x_{h r}\right)
$$

Here is the description of $\operatorname{End}\left(B_{p}\right)$ as a quotient of the matrix ring $M_{p}$

Theorem 3. (See also [3]) The map $\theta: M_{p} \rightarrow \operatorname{End}\left(B_{p}\right)$ given by $\theta\left(M_{p}\right)\left(\alpha_{11}, \ldots, \alpha_{h r}\right)^{T}=\theta\left(M_{p}\left(\alpha_{11}, \ldots, \alpha_{h r}\right)^{T}\right)$ is a surjective ring homomorphism.

Lemma 3. Let $K$ be the set of matrices $M=\left(a_{i j}\right) \in B_{p}$ such that $p \mid a_{i j}$ $\forall i, j$. Then, $K$ forms an ideal.

The following is a complete description of $\operatorname{Aut}\left(B_{p}\right)$

Lemma 4. An endomorphism $M^{\prime}=\theta(M)$ is an automorphism if and only if $M(\bmod p) \in G L_{h r}\left(\mathbb{Z}_{p}\right)$.

\subsection{Characteristic of $R=p^{2}$}

Proposition 4. Consider $B_{p}^{\prime}=\underbrace{\mathbb{Z}_{p} \times \ldots \times \mathbb{Z}_{p}}_{r}$ and $B_{p}=(\underbrace{\mathbb{Z}_{p} \times \ldots \times \mathbb{Z}_{p}}_{r})^{h+1}$. Then:

(i) $\operatorname{End}\left(B_{p}^{\prime}\right)=M_{r}\left(\mathbb{Z}_{p}\right)$;

(ii) $\operatorname{Aut}\left(B_{p}^{\prime}\right)=G L_{r}\left(\mathbb{Z}_{p}\right)$;

(iii) $\left|\operatorname{Aut}\left(B_{p}^{\prime}\right)\right|=\prod_{i=0}^{r-1}\left(p^{r}-p^{i}\right)$.

Proof. Similar to the case when the characteristic of $R=p$, with some slight modifications. 


\section{Main Results}

Lemma 5. Consider $R^{*}$ when the characteristic of $R=p$,

$$
B_{p}=\underbrace{\mathbb{Z}_{p}^{r} \times \mathbb{Z}_{p}^{r} \times \ldots \times \mathbb{Z}_{p}^{r}}_{h}
$$

and $M_{p}=\operatorname{End}\left(B_{p}\right)$. Then:

(i) $\operatorname{End}\left(B_{p}\right)=M_{h r}\left(\mathbb{Z}_{p}\right)$

$$
=\left(\begin{array}{cccccccc}
a_{11} & \cdots & a_{1 r} & a_{1(r+1)} & \cdots & a_{1(2 r)} & \cdots & a_{1(h r)} \\
a_{21} & \cdots & a_{2 r} & a_{2(r+1)} & \cdots & a_{2(2 r)} & \cdots & a_{2(h r)} \\
\vdots & \cdots & \vdots & \vdots & \vdots & \vdots & \vdots & \vdots \\
a_{r 1} & \cdots & a_{r r} & a_{r(r+1)} & \cdots & a_{r(2 r)} & \cdots & a_{r(h r)} \\
\vdots & \cdots & \vdots & \vdots & \vdots & \vdots & \vdots & \vdots \\
a_{(2 r) 1} & \cdots & a_{(2 r) r} & a_{(2 r)(r+1)} & \cdots & a_{(2 r)(2 r)} & \cdots & a_{(2 r)(h r)} \\
\vdots & \cdots & \vdots & \vdots & \vdots & \vdots & \vdots & \vdots \\
a_{(h r) 1} & \cdots & a_{(h r) r} & a_{(h r)(r+1)} & \cdots & a_{(h r)(2 r)} & \cdots & a_{(h r)(h r)}
\end{array}\right) ;
$$

(ii) $\operatorname{Aut}\left(B_{p}\right)=G L_{h r}\left(\mathbb{Z}_{p}\right)$;

(iii) $\left|\operatorname{Aut}\left(B_{p}\right)\right|=\prod_{i=0}^{(h r)-1}\left(p^{h r}-p^{i}\right)$.

Proof. Using our characterization and appealing to Hillar and Rhea's account in [3], we find all the elements of $G L_{h r}\left(\mathbb{Z}_{p}\right)$ that can be extended to a matrix in $\operatorname{End}\left(B_{p}\right)$ and calculate the distinct ways of extending such an element to an endomorphism. So, we need all such matrices $M_{h r} \in \operatorname{End}\left(B_{p}\right)$ that are invertible modulo $p$.

Now, recall that $B_{p}=\left(\mathbb{Z}_{p}^{r}\right)^{h}$ and define the following numbers:

$$
d_{h r}=\max \left\{\eta: e_{\eta}=e_{h r}\right\}, c_{h r}=\min \left\{\eta: e_{\eta}=e_{h r}\right\} .
$$

Since $e_{\eta}=e_{h r}$ for $\eta=h r$, we have the two inequalities $d_{h r} \geq h r$ and $c_{h r} \leq h r$. But in this case, $e_{1}=e_{2}=\ldots=e_{h r}$. Therefore, $d_{h r}=h r$ and $c_{h r}=i, \forall i=$ $1, \ldots, h r$. 
Thus $M_{h r} \in \operatorname{End}\left(B_{p}\right)$ are upper block triangular matrices of the form

$$
\left(\begin{array}{cccccccc}
\eta_{1_{d_{11}}} & \cdots & \eta_{1 r} & \eta_{1(r+1)} & \cdots & \eta_{1(2 r)} & \cdots & \eta_{1(h r)} \\
\eta_{21} & \cdots & \eta_{2 r} & \eta_{2(r+1)} & \cdots & \eta_{2(2 r)} & \cdots & \eta_{2(h r)} \\
\vdots & \cdots & \vdots & \vdots & \vdots & \vdots & \vdots & \vdots \\
\eta_{r d_{1} 1} & \cdots & \eta_{r r} & \eta_{r(r+1)} & \cdots & \eta_{r(2 r)} & \cdots & \eta_{r(h r)} \\
\vdots & \ddots & \vdots & \vdots & \vdots & \vdots & \vdots & \vdots \\
& & & \eta_{(2 r)(r+1)} & \cdots & \eta_{(2 r)(2 r)} & \cdots & \eta_{(2 r)\left(d_{h r}\right)} \\
& & & & \ddots & & & \vdots \\
0 & & & & & & \ddots & \eta_{(h r)\left(d_{h r}\right)}
\end{array}\right)
$$

The number of such $M_{h r}$ is $\prod_{i=0}^{(h r)-1}\left(p^{h r}-p^{i}\right)$ since we require linearly independent columns. So, the first step for calculating $\left|A u t\left(B_{p}\right)\right|$ is done.

Next, to extend each entry $\eta_{i j}$ from $\eta_{i j} \in\left(\mathbb{Z}_{p^{e_{i}}}^{r}\right)^{h}$ to $a_{i j} \in\left(\mathbb{Z}_{p^{e_{i}}}^{r}\right)^{h}$ such that $a_{i j} \equiv \eta_{i j}(\bmod p)$, since $e_{1}=e_{2}=\ldots=e_{h r}=1, \forall i, j$, we have $p^{e_{i}}$ ways to do so for the necessary zeros (That is, when $e_{i}>e_{j}$ ). Similarly, there are $p^{e_{i}-1}$ ways for the not necessarily zero entries, that is, when $e_{i}=e_{j}$ as any element of $\left(\mathbb{Z}_{p^{e_{i}}}^{r}\right)^{h}$ works.

Lemma 6. Consider $R^{*}$ when the characteristic of $R=p^{2}$,

$$
B_{p}=\underbrace{\mathbb{Z}_{p}^{r} \times \mathbb{Z}_{p}^{r} \times \ldots \times \mathbb{Z}_{p}^{r}}_{h+1}
$$

and $M_{p}=\operatorname{End}\left(B_{p}\right)$. Then:

(i) $\operatorname{End}\left(B_{p}\right)=M_{(h+1) r}\left(\mathbb{Z}_{p}\right)=$

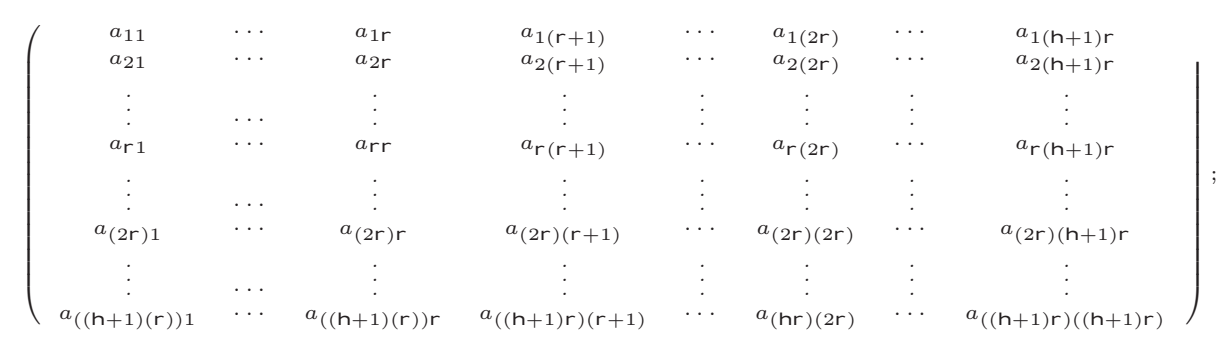

(ii) $\operatorname{Aut}\left(B_{p}\right)=G L_{(h+1) r}\left(\mathbb{Z}_{p}\right)$;

(iii) $\left|\operatorname{Aut}\left(B_{p}\right)\right|=\prod_{i=0}^{((h+1) r)-1}\left(p^{(h+1) r}-p^{i}\right)$. 
Proof. Follows from the proof of the previous lemma with some slight modifications.

Theorem 4. Let $R^{*}$ be the unit group of a class of finite rings described by the construction in Section 1. Then:

(i) When the characteristic of $R=p, \operatorname{Aut}\left(R^{*}\right)=\operatorname{Aut}\left(\mathbb{Z}_{p^{r}}-1\right) \times \operatorname{Aut}\left(B_{p}\right)$,

$$
\left|\operatorname{Aut}\left(R^{*}\right)\right|=\left|\mathbb{Z}_{p^{r}}^{*}-1\right| \times\left|\operatorname{Aut}\left(B_{p}\right)\right|=\Phi\left(p^{r}-1\right) \cdot \prod_{i=0}^{(h r)-1}\left(p^{h r}-p^{i}\right)
$$

(ii) When the characteristic of $R=p^{2}$, then

$$
\left|\operatorname{Aut}\left(R^{*}\right)\right|=\Phi\left(p^{r}-1\right) \cdot \prod_{i=0}^{((h+1) r)-1}\left(p^{(h+1) r}-p^{i}\right) .
$$

\section{Acknowledgements}

The first author acknowledges the co-authors for their meaningful and well done $\mathrm{PhD}$ supervision. Their selflessness in giving scholarly comments and direction is invaluable. May God bless you. A lot of thanks to Masinde Muliro University of Science and Technology for their financial support towards my research stay at the University of Kwazulu Natal, South Africa.

\section{References}

[1] A. Ranum, The group of classes of congruent matrices with application to the group of isomorphisms of any Abelian groups, Trans. Amer. Math. Soc., 8 (1907), 71-91, doi: 10.1090/S0002-9947-1907-1500775-1.

[2] C.J. Chikunji, On unit groups of completely primary finite rings, Mathematical Journal of Okayama University, 50 (2008), 149-160.

[3] C. Hillar, D. Rhea, Automorphisms of an Abelian p-group, Amer. Math. Monthly, 114 (2007), 917-922.

[4] K. Shoda, Uber die Automorphismen einer endlichen Abelschen Gruppe, Math. Ann., 100 (1928), 674-686, doi: 10.1007/BF01448871.

[5] M.O. Oduor, M.O. Ojiema, M. Eliud, Units of commutative completely primary finite rings of characteristic $p^{n}$, International Journ. of Algebra, 7, No. 6 (2013), 259-266.

[6] Y. Alkhamees, Finite Rings in which the multiplication of any two zero divisors is zero, Arch. Math., 37 (1981), 144-149, doi: 10.1007/BF01234337. 